\title{
Orodispersible Films: Towards Drug Delivery in Special Populations
}

Mariagiovanna Scarpa ${ }^{1}$, Sven Stegemann ${ }^{2}$, Wen-Kai Hsiao ${ }^{3}$, Heinz Pichler ${ }^{3}$, Simon Gaisford $^{1}$, Massimo Bresciani ${ }^{3}$, Amrit Paudel $^{2,3, *}$, Mine Orlu $^{1}$

${ }^{1}$ School of Pharmacy, University College London (UCL), London, United Kingdom

${ }^{2}$ Graz University of Technology, Graz, Austria

${ }^{3}$ Research Center Pharmaceutical Engineering GmbH, Graz, Austria

*Corresponding author: Email: amrit.paudel@rcpe.at

Research Center Pharmaceutical Engineering, Inffeldgasse 13, 8010, Graz, Austria

Tel.: +43 (316) 873 - 30912; Fax: +43 (0) 316 /873-10-30912 


\begin{abstract}
Orodispersible films (ODF) hold promise as a novel delivery method, with the potential to deliver tailored therapies to different patient populations. Together with the current trends and potential therapeutic areas such technology can address, some of the unmet needs in terms of quality aspects and manufacturing of conventional and novel (printed) ODF are discussed. Overall, this article aims to stimulate further research to fill the current knowledge gap between manufacturing and administration requirements of ODF targeting specific patient subpopulations such as geriatrics.
\end{abstract}

Keywords: orodispersible film, inkjet printing, acceptability, substrate, disintegration, dropon-demand 


\section{ODF: A potential formulation strategy for special patient populations}

There is an increasing need of developing new drug delivery platforms to address the needs of special patient populations. For example, the paediatric patient requires the medicine dosage to be adequate to the constantly increasing body weight. As medicinal products containing the required dosage may not be available, the practice of splitting tablets, opening capsules, or mixing powders with foods or liquids may lead to dose inaccuracy and other consequences for the safety of the patient and the efficacy of the treatment (Visser et al., 2016). Similarly, other populations may have specific needs with respect to medicine administration (Grimsrud et al., 2015; Slavkova and Breitkreutz, 2015; Stegemann et al., 2010). Therefore, the development of drug delivery platforms that can help addressing the needs of specific patient populations is greatly needed.

Orodispersible films have been reported to contribute to an improved patient compliance by improving ease of administration and by not requiring water (Bala et al., 2013; Krampe et al., 2015). Furthermore, ODFs offer a wide range of features that can be easily adapted to the needs of individual patients or patient populations.

According to Borges and colleagues, patients suffering from dysphagia or subjected to an increased risk of chocking can benefit from the employment of ODF platforms for drug administration. As ODFs are inherently easy to administer, young and older patients can have access to a platform that can ensure a complete and precise dosing of medicines, minimising the risk of partial loss of actives due to tablet crushing or imprecise liquid administration. Moreover, medicine administration to uncooperative patients can be facilitated by the adhesion of ODFs to the oral cavity, therefore preventing the medicine to be spit out. Other ODF features such as flexibility, portability, and stability can confer superiority to other dosage forms like orodispersible tablets or liquids (Borges et al., 2015a) (Borges at al., 2015 vol I). Delivering actives via ODFs through oral mucosa, can lead to rapid dissolution and 
fastest onset of action (Ashraf and Sayeed, 2014; Choudhary et al., 2013). Some examples of ODF products are commercially available for Alzheimer's disease (Donepezil - Labtech, Kyukyu Pharmaceuticals, Hexal Pharmaceuticals), emesis (Ondansetron - Monosol, Labtech, Aavishkar), schizophrenia (Olanzapine - Labtech, Hexal Pharmaceuticals), migraine (Zolmitriptan - Monosol, Labtech, NAL Pharma) etc. (Borges et al., 2015a). The readers are referred to a recent review by Borges et al. for a comprehensive product list of oro-mucosal film based products that are registered, launched, under (pre-) clinical development or withdrawn/ discontinued.

The acceptability of ODFs was described in a recent paper considering the features of the final product including appearance, composition, taste and mouthfeel (Krampe et al., 2015). ODFs are considered to be age-appropriate oral dosage forms mainly with respect to its disintegration in the oral cavity upon administration without water. Furthermore, ODFs offer the possibility to implement taste masking technologies in order to minimise the averseness of the taste of the Active Pharmaceutical Ingredient (API). Mouthfeel and texture are also considered key characteristics potentially affecting the acceptability of ODFs, particularly with regards to the presence of residual particles following disintegration (Krampe et al., 2015). Mouthfeel in the sensory evaluation of foods has been related to the primary role of the saliva function. A reduced salivary production could therefore alter the mouthfeel of a product in specific patient groups such as patients under treatment for HIV, or older patients subjected to polypharmacy (Gupta et al., 2006; López-Verdín et al., 2013; Stokes et al., 2013). Other features like appearance, mucosal irritation, and API absorption are also considered relevant features to take into consideration when designing an ODF formulation (Krampe et al., 2015).

In addition to the aforementioned ODF characteristics, other features could potentially positively or negatively affect the patient acceptability of the dosage form, and should be 
further explored. As proposed in Figure 1, the handling, placement and disintegration of ODFs are key stages to identify the full product features with high potential impact on patient experience. For example, the "stickiness/adhesiveness" of the film potentially contributes to placement in the mouth and subsequently the overall mouthfeel of the product. Krampe and colleagues have referred to the "gummy" nature of the films after wetting as potentially contributing to the mouthfeel of the dosage form (Krampe et al., 2015). Moreover, in the case of patients experiencing poor manual dexterity, poor hand sensitivity or reduced pinch strength (Stegemann et al., 2016), high ODF stickiness may result in the inability to properly handle the dosage form. These properties are largely related to the intrinsic properties of the carrier polymers, eg. hygroscopicity and interfacial attributes. The ODF formulation is designed to disintegrate fast once placed in the mouth. Wettability, disintegration, and dissolution time of the film may change depending on the saliva production rate of the user. These parameters are considered to govern the performance of ODF drug products. Therefore, in the case of patients affected by severely impaired saliva production (dry mouth syndrome), ODFs may not be the dosage form of choice for drug administration. 


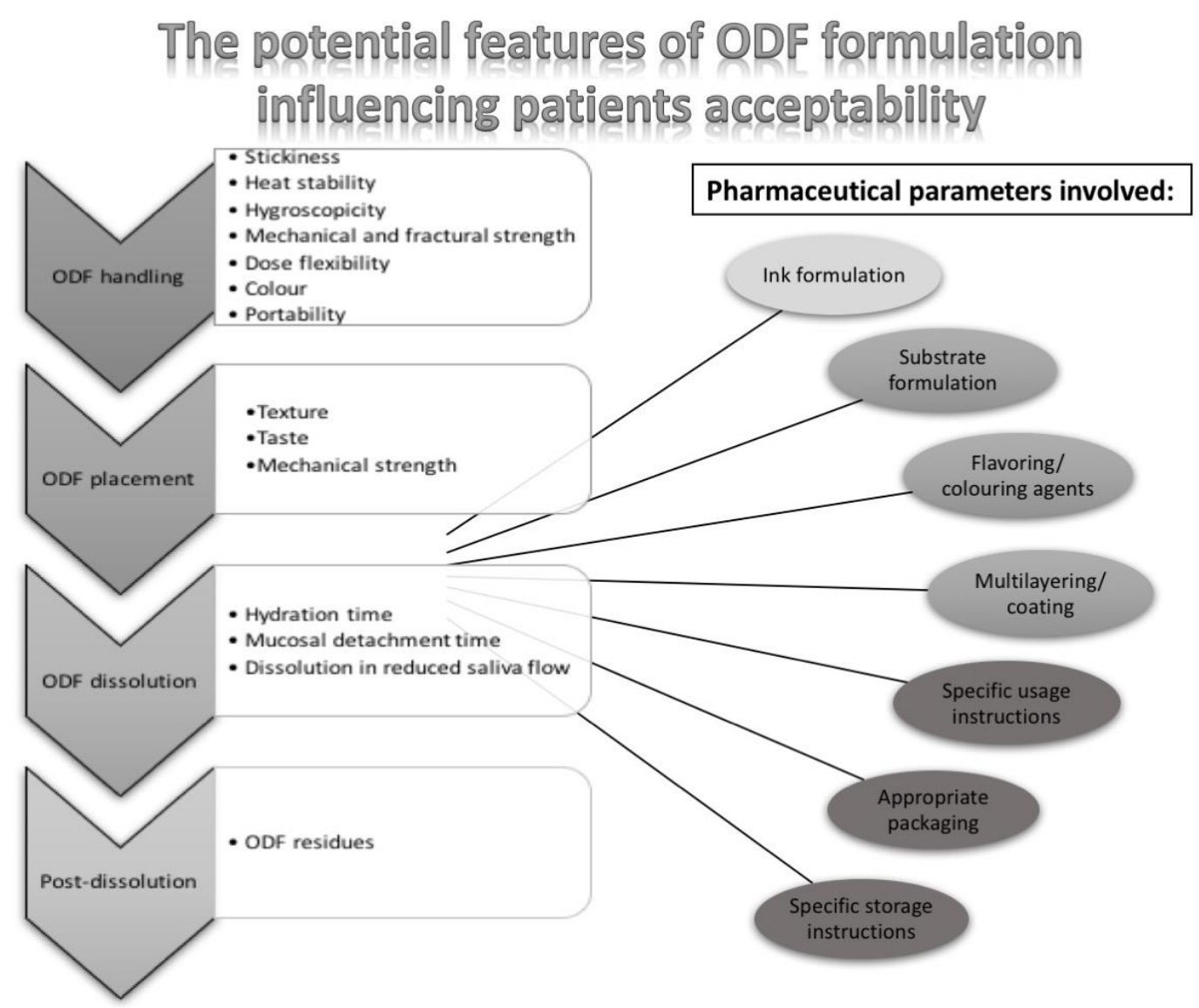

Figure 1: The potential features of ODF formulation influencing different criteria of films as potential patient acceptability factors (Ink formulations in case of printed ODF, described below).

\section{Formulation, process and quality considerations of orodispersible films products}

ODF products are conventionally manufactured via film casting of solution, suspension or melt using diverse technologies such as solvent casting, semisolid casting, rolling, coating and hot melt sheet extrusion (Borges et al., 2015a; Krampe et al., 2015). In these cases, the API(s) is/are dispersed in a feedstock with the suitable excipients, formulation and/or process aids and processed to yield films of desired dimensions and mechanical properties. Typically, therefore, ODF formulations have the structure of a matrix-based composite film. The current co-matrix formulation and process platforms intrinsically harbour some 
limitations in terms of content uniformity and the ability to combine and diversify doses because the final formulation is homogeneous. Furthermore, due to their moderate size and thickness ODFs can typically be loaded only with limited drug concentrations per unit volume and surface area (Bala et al., 2013). Therefore, oral films are not generally suitable for treatments requiring high dosages, although up to 50\% drug loading in an ODF (Gas-X Strips ${ }^{\circledR}$, Novartis) has been reported (Siddiqui et al., 2010).

The ODF design process should take several parameters into consideration: therapeutic target, patient population, safety, appropriateness and compatibility of API and excipients, processability of the polymeric mixture, impact on stability, physicochemical and mechanical characteristics of the final product, residual solvent/water content, drug release profile, packaging, and acceptability to the end user. Each pharmaceutical development stage can have a deep impact on the quality of the final product and must be carefully evaluated.

\subsection{Manufacturing technologies}

The most widespread technologies for ODF manufacture include solvent casting and semisolid casting as broadly described by Hoffmann and colleagues (Hoffmann et al., 2011a). These generally include feed stock of drug-excipient solution or suspension in aqueous and/or non-aqueous media. The use of organic solvents in solvent casting can improve the solubility of some APIs. Conversely, residual amounts of solvents could remain in the final product. Other manufacturing parameters, such as casting solution homogeneity, stability and film thickness, must be accurately monitored to ensure a smooth process.

Hot melt extrusion (HME) offers the advantage of a solvent-free process for ODF manufacturing (Palem et al., 2016, 2013; Park et al., 2015; Repka et al., 2003). Yet, poor availability of suitable polymers and high temperatures may pose stability issues to the active (Hoffmann et al., 2011). HME is a continuous manufacturing process. The scaling up/ down for ODF manufacturing via hot melt sheet/film extrusion can depend upon the capacity of 
downstream shaping process. Other recently developed technologies offer alternatives to ODF manufacturing. Electrospinning of drug-loaded polymeric solutions has increased in popularity in the pharmaceutical area. This technology allows an increase in drug solubility and produces fast dissolving polymeric films in a cost-effective way. Recently, drug loaded electrospun fast dissolving nanofibers have been manufactured and characterised (Illangakoon et al., 2014). The key challenges for electrospun fibers are their poor mechanical characteristics and poor handling characteristics that can impede downstream manufacturing (filling, flow, packaging) as well as intake by patient.

Establishment of dedicated facilities for development and commercial scale production of personalised ODFs requires many techno-economic considerations (Rantanen and Khinast, 2015; Tucker et al., 2016). Some of them are: i) availability of manufacturing technologies and status of in terms of readiness for the industrialisation, ii) types of equipment, scale and cleaning/maintenance and possibility of scale up and scale down, iii) (critical) process parameters, process integration, control and monitoring, iv) batch versus continuous operations, v) throughput/capacity and vi) packaging. The variation in formulations (eg. water content of the feed, viscosity, melt temperature) is the one of the key determinants of cooling/ drying time and therefore the entire process efficiency. On the other hand, the required size of the strip varies with the (personalised) dose, which can complicate primary packaging (eg. different pouch sizes and sealing times).

\subsection{General formulation principles}

The basic structure of oral films is a polymeric matrix. Typical polymeric carriers used in ODF manufacturing include cellulose, starches, gums and polysaccharides, proteins, and semi-synthetic or synthetic pharmaceutical grade polymers (Borges et al., 2015b). Carrier polymer selection prerequisites for ODF formulations include solubility in water and/or diverse processing solvents, film forming properties, compatibility with wide range of APIs, 
safety and availability of different molecular weights/ degrees of substitution (Moritz, 2006). Depending on the physical and chemical properties of polymers or mixtures of polymers and the quality target product profile, the processability can be either favoured or impeded. For example, highly viscous polymer mixtures in melt extrusion lead to difficulty in processing. In this respect, it is common practice to incorporate some plasticisers in the formulation. In addition surfactants are used to enhance solubility of poorly soluble drugs as well as the wettabilityof ODFs (Hoffmann et al., 2011a).

Some small fractions of other functional excipients such as humectants and/or saliva stimulating agents, sweeteners, oro-mucosal permeation enhancers and trace colouring/flavouring agents are used (Hoffmann et al., 2011a). The impact of each component to the final product should be evaluated during development. Therefore, the selection of film forming polymers should result in a balanced choice ensuring good processability yet possessing the desired film characteristics, most importantly in terms of in vitro and in vivo pharmaceutical performance and stability.

\subsection{Quality aspects of the final product}

In the absence of regulatory guidelines and due to the novelty of the dosage form, typical film potential Critical Quality Attributes (pCQA) have been described in recent publications (Borges et al., 2015a; Irfan et al., 2015). Oral films should have appropriate mechanical strength to endure both the stress of the manufacturing pipeline, and folding during postproduction, while maintaining the targeted disintegration and drug release profile. Mechanical characterisation studies should include measurement of thickness, tensile strength, elongation at break, tear resistance, folding endurance, and Young's modulus (Irfan et al., 2015). Stability during all manufacturing stages must be carefully studied beforehand and monitored throughout. Particular attention should be paid to unintended or uncontrolled 
chemical reactions between active and excipients, physical property modifications due to high temperatures, and interactions between films and packaging material.

The drug release profile is another fundamental quality aspect for all types of orally administered films. How fast the film disintegrates, and the rate at which the drug is being released classifies the type of film being produced. With respect to disintegration and dissolution characterisation, a range of different dissolution/disintegration testing is available, although there is currently no harmonised approach towards the utilisation of a standard methodology. Due to the lack of specific guidelines, USP 1 or 2 dissolution apparatus is currently used as standard assays, although several criticisms have been made to the high volumes of dissolution/disintegration media employed (Garsuch and Breitkreutz, 2010). Film disintegration and dissolution largely depend on the film-forming polymer being used as well as the physicochemical properties of API. The residual water remaining at the end of the manufacturing process can significantly affect the mechanical properties, tackiness, adhesion properties and stability of the final product. Humidity of the manufacturing site should therefore be controlled and appropriate packaging provided. The organoleptic properties of films are mainly evaluated based on their taste. Specific flavours and taste masking technologies can be used to target different patient populations. Finally, dose uniformity, batch uniformity, homogeneity, impurity profile are likely to be some of the most challenging aspects to control. In addition, the physical form of the active and excipients (polymorphic form, amorphicity, hydrate/solvate) and phase homogeneity (miscibility, molecular mobility etc.) are crucial in terms of drug release characteristics as well as physical and chemical stability, therefore should be duly considered. Finally, microbiological characterisation can be a quality requirement of ODFs as most of these films are prepared from aqueous feed and nutritional carbohydrate or cellulosic polymers. 
The development of in-line Process Analytical Technology (PAT) tools adaptable for monitoring and control of technologies for personalised/individualised drug product (ODF) manufacturing is one of the growing areas of interest for both industry and academia. Zhang and colleagues recently demonstrated the implementation of Raman spectroscopy as the in line and off line tool for quantification of poorly soluble drugs in strip films (Zhang et al., 2014).

\section{Inkjet printing as an emerging population centric ODF product manufacturing platform}

\subsection{General overview}

Population-based manufacturing platforms should provide dose flexibility and allow for fixed dose combinations to adjust to physiological condition, co-morbidity and altered pharmacokinetics and pharmacodynamics. The conventional cast films offer dose stratification mainly based on the cut-strip size, thickness (Krampe et al., 2015), and weightstrip size. This complicates possible cross- or downstream processes as well as primary packaging. Two dimensional printing of liquid API or formulation (as inks) to solidify in and/or on to ODF as substrate holds considerable potential in flexible dose manufacturing with minimal process-steps. Furthermore, InkJet-printed-ODFs (IJ-ODFs) could provide dose flexibility through the adjustment the volume of liquid API deposited onto a fixed-size substrate rather than adjusting the size of the film via cutting into different sizes as for conventional cast films. Furthermore, homogeneity of drug distribution in cast-films for low dose and/or poorly soluble actives is intrinsically challenging. In this respect, inkjet printing technology onto and/or into ODFs has been considered in recent years as a flexible manufacturing opportunity for personalised ODF manufacture (Cheow et al., 2015; Genina et al., 2013b; Sandler et al., 2011; Vakili et al., 2015). Current studies are being conducted on 
the application of the inkjet printing technology to produce dose-flexible oral films (Alomari et al., 2014). A wide diversity of finished solid oral dosage forms such as tablets, hard/soft gelation capsules can act as the substrate for printing (Boehm et al., 2015, 2014). For example, the surrogate technology of dispensing drug solutions or suspensions into placebo tablets was developed and readied for commercial manufacturing by GlaxoSmithKline (GSK) for highly potent actives (Clarke et al., 2012). Since ODFs are the topic of this article, discussion in this direction has been excluded.

Precise and accurate dispensing of very small doses (pico to micro liter) is achievable by printing technology. Small volumes of drugs in liquid can be dispensed on-demand onto the film surface to ensure a precise therapeutic drug dose to be delivered to the patient. Inkjet printing technologies, resolution/ accuracy, scale, throughput, speed etc. have significantly evolved over the last decade (Derby, 2010; Sowade et al., 2016).

Inkjet printing technology could be also used for pharmaceutical printing of multiple drugs onto a single substrate, ensuring drug compatibility, dose precision, and multiple medicationrelated issues (Preis et al., 2015). The therapeutic areas where inkjet-printed orodispersible film (IJ-ODFs) can potentially improve the ease of administration include pathologies where the patient experiences difficulty in self-medicating (severe psychological and neurological disorders), and/or difficulty in swallowing (severe neurodegenerative disorders causing swallowing difficulties). Therefore, uncooperative/ cognitively impaired, and dysphagic patients are major populations that can benefit from rapidly dissolving IJ-ODFs (Abdelbary et al., 2014). Furthermore, fast drug-releasing IJ-ODFs design can be further explored for managing emergency conditions that require rapid onset of action of the administered drug (Karki et al., 2016), such as anaphylactic shock, congestive heart failure and sudden epileptic seizure, breakthrough pain and so on (Alayoubi et al., 2016). The superiority of the drug release performance and medication efficiency of IJ-ODFs against traditional (pre) drug- 
loaded ODF is subject to further verification. IJ-ODFs, currently under development, can better address conditions requiring high dosage precision (Peck, 2015), fixed dose combinations (FDC), by printing physicochemically interacting actives and excipients spatially as separate drops, and low dosage treatments (Bala et al., 2013; Preis et al., 2015). Regulation of drop size, printing pattern and density, etc. can modify the concentration of drug printed on a single film with a very high precision (Singh et al., 2010). Further, the opportunity of printing specific patterns could serve as a useful form of identification of the specific medicinal preparation, especially for patients subjected to polypharmacy (Preis et al., 2015). The technology is still in a developing phase and readying towards compliance to the Good Manufacturing Practice (GMP) requirements for commercial-scale personalised pharmaceutical dosage manufacturing (Nallan et al., 2014). More precisely, selection of printer material compatible with printing ink, development of cleaning and process validation protocols are important issues to be addressed. Similarly to the more recent 3D printing technology (Ventola, 2014), the Inkjet printing platform could find applicability in the pharmacy setting. A personalised orodispersible film can be printed on-site by the pharmacist in order to respond to the needs of the individual patient (Alomari et al., 2014; Lind et al., 2016). For instance, the required therapeutic dose can be adjusted on-site in particular benefitting paediatric patients.

\subsection{Inkjet printing on ODFs: A case study}

An exciting advancement in developing understanding of actives printed onto ODFs to produce personalised dosage forms has been described in a recent publication (Planchette et al., 2015). A non-contact printing system that incorporates complementary inkjet technologies (piezoelectric actuator or solenoid valve) was utilised (Figure 2). Based on this approach, a wide range of inks including solutions of physicochemically diverse APIs, polymeric nano-suspensions and excipient coating solutions were successfully printed. The 
printing process of these diverse inks onto different types of commercial oral ODF placebos and customised films were optimised to avoid substrate damage (defects, holes, tears). Morphological analysis of the printed surface clearly showed dependence on the ODF type used (Figure 3). A consistent dispensing of drugs with a volumetric deviation of less than \pm 2 $\%$ has also been achieved.

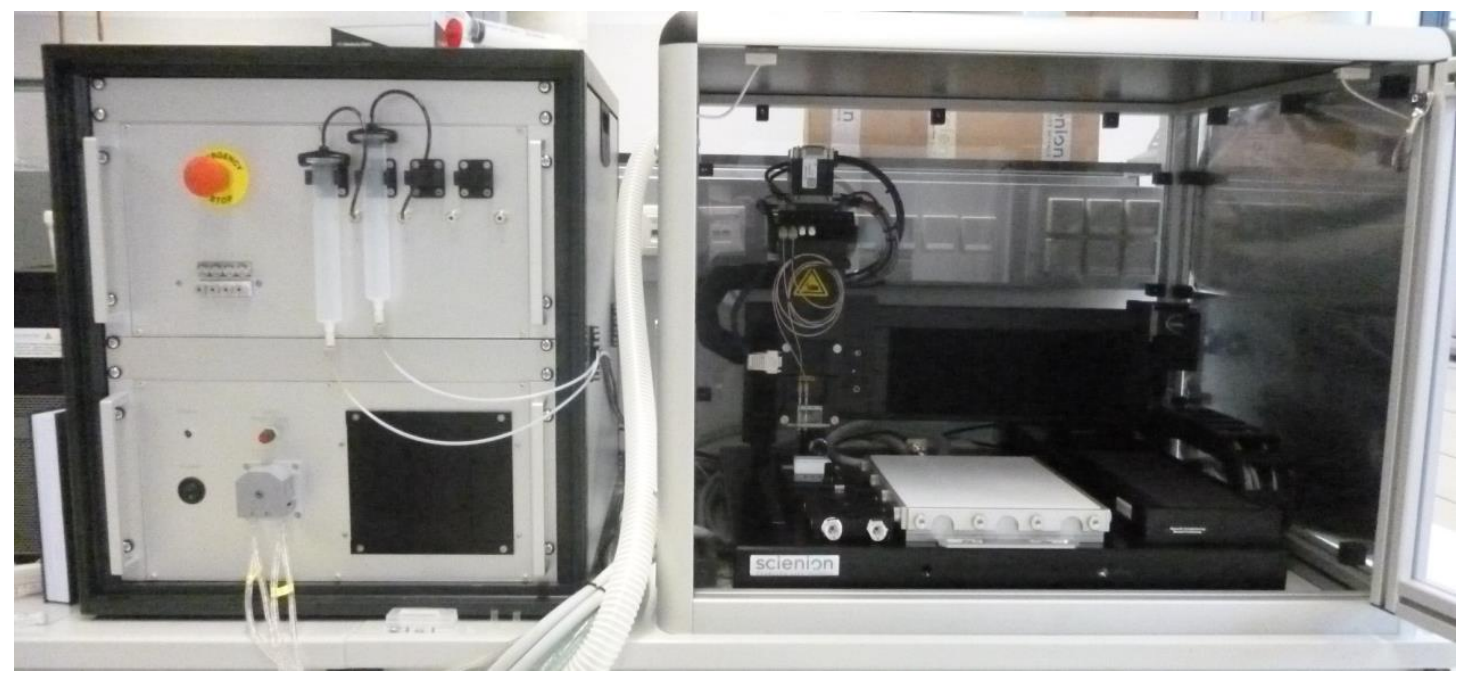

Figure 2: Non-contact Printing System SciFLEXARRAYER S3 (Scienion AG, Berlin, Germany) incorporating two complementary ink jet technologies.

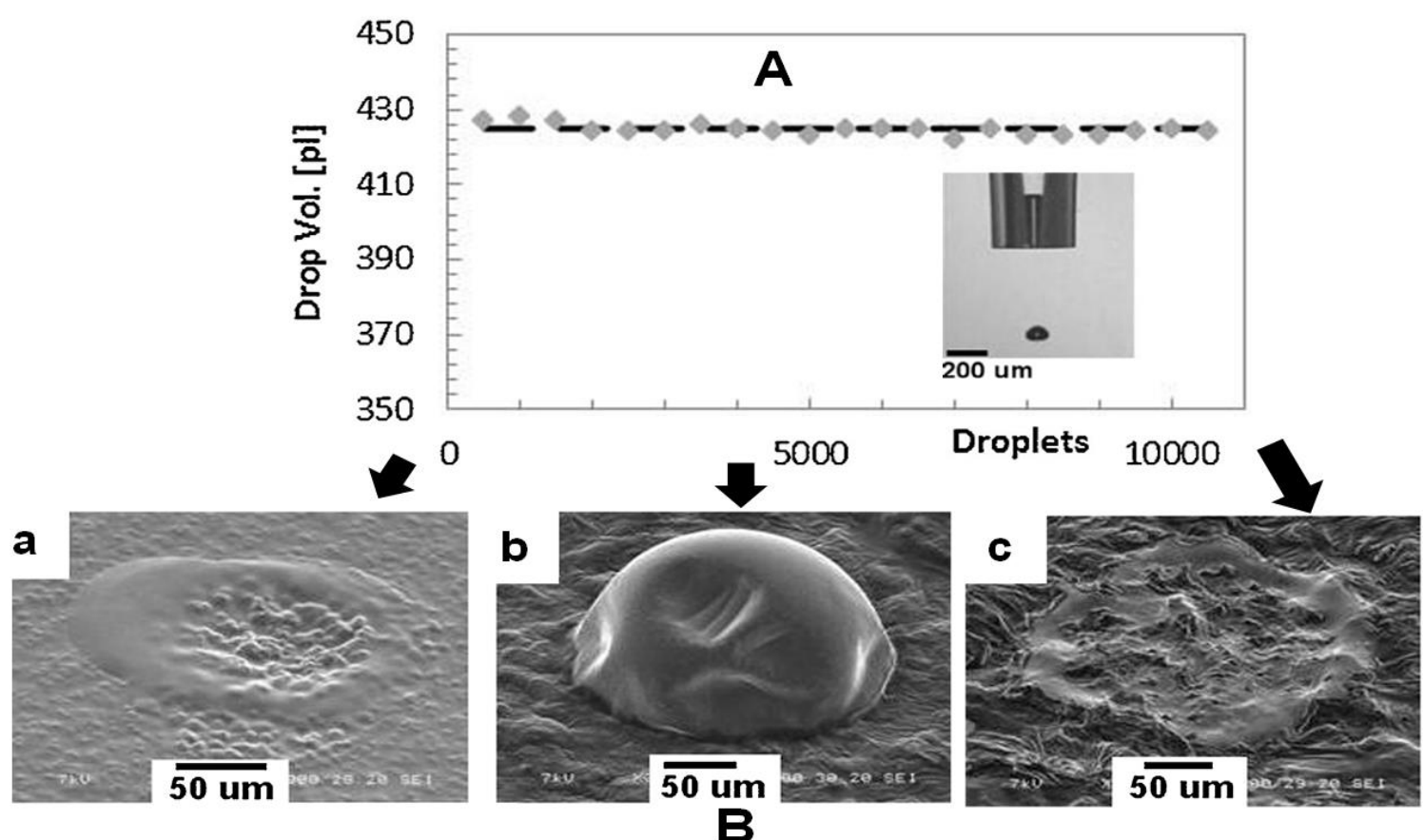

Figure 3: An overview of inkjet printing of sodium picosulfate solution on different ODF substrate. (A) Plot of drop volume measured by drop watcher $(\diamond)$ and that calculated based on HPLC analysis (solid line) with the picture of dispensing unit (inset) and (B) SEM images of printed droplet on to (a) Rapidfilm ${ }^{\circledR}$, (b) hydrophobic non-porous film and (c) hydrophilic porous film. Reprinted (adapted) with permission from (Planchette et al., 2015) Copyright (2015) Elsevier. 


\subsection{Inkjet printing for personalised ODF manufacturing: unmet needs}

Inkjet printing technology definitely requires further scientific efforts before the pharmaceutical drug delivery community embraces personalised dosage manufacturing. Engineering solutions to allow the diverse range of benchtop printers, currently used in research and development $(\mathrm{R} \& \mathrm{D})$ applications, up to GMP compliant industrial scale processing is the biggest challenge to successful pharmaceutical implementation. Formulation aids (e.g. surfactants, crystallisation inhibitors, preservatives etc.) and process aids (e.g. viscosity modifiers, co-solvents etc.) can improve the specific printability parameters of drug-containing ink solutions such as surface tension and viscosity as well as final properties of the printed dosage forms (drug distribution and stability) (Di Risio and Yan, 2007; Genina et al., 2012; Wickström et al., 2015). For sufficient loading of poorly soluble drugs, the ink formulations needs to be modified accordingly (non-aqueous solutions, nano-suspensions, lipidic formulations) (Williams et al., 2013). Nucleation and crystal growth, Ostwald ripening in supersaturated solutions/ suspensions of poorly soluble drug can commence during feeding or during dispensing (nozzle tips). Inkjet printing using excipient stabilised aqueous nano-crystalline APIs as an ink and ODF as a substrate is currently sought after as a viable alternative to fabricate individualised dosage form of poorly soluble drug (Cheow et al., 2015; Hilgert et al., 2014).

Beyond ink formulation, various inkjet printing related factors such as satellite drops, nozzle clogging, fast evaporation of volatile solvents, coffee ring effect have been an ongoing academic and industry R\&D topic (Deegan et al., 1997; Hoath et al., 2008, 2012; Reis et al., 2005; Stringer and Derby, 2010). For example, an empirical effort to validate the regime of fluid "jettability" has been described by Kuang and colleagues in 2014 (Nallan et al., 2014). Now, the challenge is to translate this fundamental understanding towards advancing IJ-ODF platforms. Figure 4 illustrates potential ink formulation parameters and printing process 
parameters that can be critical in obtaining the desired quality attributes of the printed ODF dosage forms.

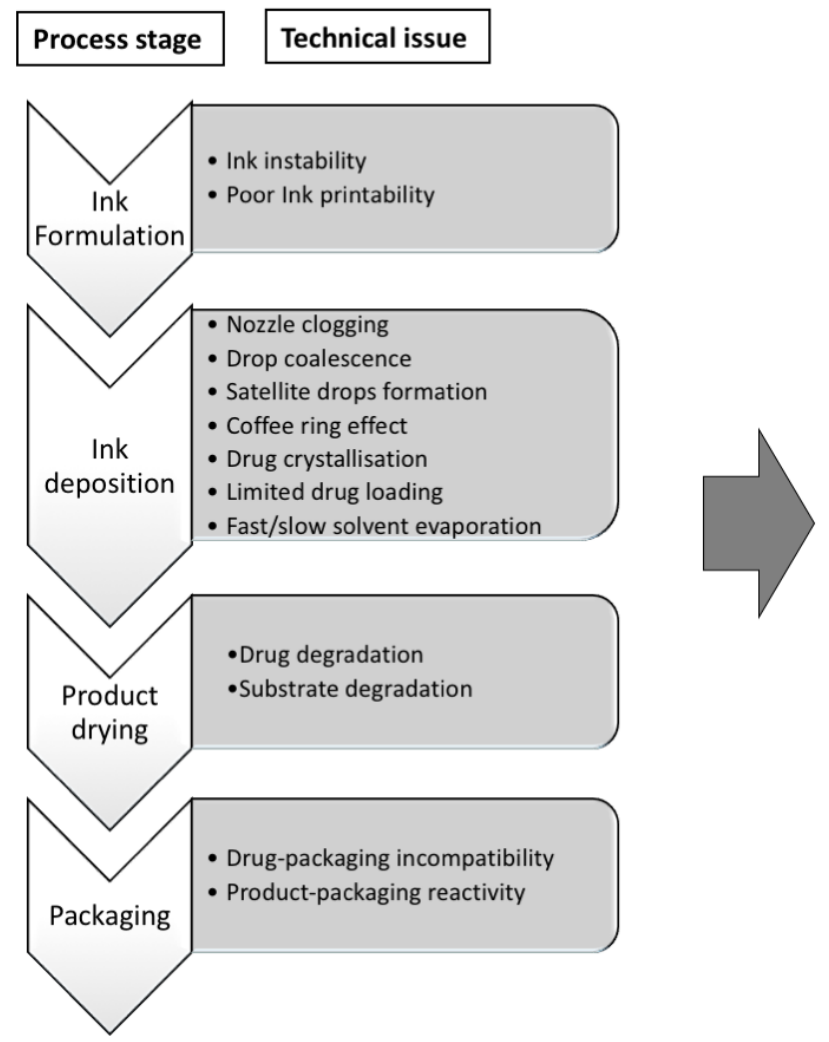

\begin{tabular}{|l|}
\multicolumn{1}{|c|}{ Technical parameters modification } \\
\hline Adoption of different ejection system \\
\hline Change of printer resolution \\
\hline Change of pattern resolution \\
\hline Adjustment of nozzle distance \\
\hline Change of drop frequency \\
\hline Adjustment of printability parameters \\
\hline Adjustment of solvent evaporation rate \\
\hline Change of ink temperature \\
\hline Change of ink viscosity \\
\hline Modification of ink formulation \\
\hline Check of substrate-ink compatibility \\
\hline Change of substrate temperature \\
\hline Modification of substrate formulation \\
\hline Deposition of substrate multilayers \\
\hline Deposition of coating layer \\
\hline Change of packaging material \\
\hline
\end{tabular}

Figure 4: Technical issues involved in various stages of the inkjet printing process and associated parameters amenable for process/ product optimisation.

\subsection{Importance of ODF substrate for inkjet printing}

Following the optimisation of ink formulation and printing process, the substrate properties are of utmost importance to meet the requirements for desirable product properties (Daly et al., 2015). ODFs as a printing substrate should be developed with consideration given to incompatibilities with the potential ink components (drug, solvent, additives) (Genina et al., 2013; Preis et al., 2015; Raijada et al., 2013). Poorly soluble drugs are normally challenging to incorporate in a water-soluble substrate without sufficient molecular miscibility and interfacial interactions. Nucleation and crystal growth on thin films are dominated by surface mobility with much faster kinetics than in bulk (Soutari et al., 2012). Further studies on interfacial crystallisation will give some insight about the best strategy to adopt in order to predict and control the post-deposition of API morphology (H.-Y. Hsu et al., 2015). Along 
with substrate chemistry, modifying film microstructure (such as porosity, inter-connectivity) can be an alternatives to enhance drug loading and confine crystal growth, provided costeffectiveness and stability (Genina et al., 2013; Sandler et al., 2011).

\subsection{Product quality control and analysis}

Process monitoring and control in terms of content uniformity and compositional homogeneity in the printed dosage forms is one of the key issues to be addressed for IJ-ODF development. Model-based process development that involves real time monitoring of the deviations in droplet volume dispensed through printer can aid feedback control of the process and the time of printing. Furthermore, quantification of API in printed dots must evolve from nano- to pico-liters drops. Therefore, using classical PAT tools is challenging for real time quality control of low dose printed formulations. Recently, Palo and colleagues used Attenuated Total Reflectance-Fourier Transformed Infrared Spectroscopy (ATR-FTIR) for quantification of two APIs in the printed dosage form (Palo et al., 2016). To this end, hyperspectral (Near Infrared, Raman, Terahertz spectroscopy, Raman spectroscopy and chemical imaging etc.) imaging holds immense potential for developing the analytical method for API assay and distribution in ODF films (Vakili et al., 2015b; Edinger et al., 2017) . Novel analytical tools such as optical coherence tomography and interferometry can provide fast and non-invasive analysis of printed layer thickness, surface roughness/deformation created via solvent evaporation, the degree of adherence of printed drops with the substrate as well as spatial distributions (Sandler et al., 2014). Newman and colleagues recently employed Second Harmonic Generation (SHG) microscopy-guided synchrotron powder X-ray diffraction to detect $100 \mathrm{ppm}$ crystalline ritonavir in a hydroxypropyl methylcellulose (HPMC) matrix (Newman et al., 2015), while SHG microscopy-coupled confocal Raman spectroscopy enabled analysis of trace crystallites of paclitaxel in lyophilized Abraxane powder (Schmitt et al., 2015). Such analyses on printed 
films probably offer solid-state distribution in printed dots and their homogeneity

(Chowdhury et al., 2014).

\subsection{Inkjet printing for individualised ODF form: S.W.O.T. analysis}

Upgrading industrial-scale inkjet printing for pharmaceutical ODF manufacturing under a strictly regulated environment requires significant development effort and capital investment.

The aforementioned points and the need for dedicated downstream packaging for printed

ODFs are considered in a strengths, weaknesses, opportunities and threats (S.W.O.T.)

benchmark analysis for this platform, as presented in Table 1.

Table 1. S.W.O.T. analysis on inkjet printing for personalised IJ-ODF manufacturing.

\begin{tabular}{|c|c|}
\hline Strengths & Weaknesses \\
\hline $\begin{array}{l}\text { - Capability of precise dosing to deliver micro- to } \\
\text { nano- scale drug loading } \\
\text { - Possibility of stratified dosage manufacturing and } \\
\text { one step production of fixed dose combinations } \\
\text { (FDCs) } \\
\text { - Availability of a wide ranges of engineering } \\
\text { options and stronger technological know-how } \\
\text { existing in allied areas } \\
\text { - Flexibility of downstream processing }\end{array}$ & $\begin{array}{l}\text { - Limiting to low dose therapies due to printing } \\
\text { volume limitation and substrate drug loading } \\
\text { capacity } \\
\text { - Difficulty to achieve drop volume uniformity for } \\
\text { challenging APIs eg. poorly soluble drugs } \\
\text { - Unavailability of GMP machines for } \\
\text { pharmaceutical manufacturing } \\
\text { - Demand for process monitoring and product } \\
\text { quality control }\end{array}$ \\
\hline Opportunities & Threats \\
\hline $\begin{array}{l}\text { - Population targeted dosage form manufacturing } \\
\text { (eg. geriatric, paediatric, rare disease, micro- } \\
\text { nutrients) } \\
\text { - FDC preparations of physicochemically interacting } \\
\text { APIs } \\
\text { - Possibly superior strategy for fast releasing ODF } \\
\text { intended for emergency medications } \\
\text { - Substrate engineering to prepare printable ODFs } \\
\text { - Line extension and life cycle management for } \\
\text { pharmaceutical companies, agricultures, } \\
\text { nutraceuticals }\end{array}$ & $\begin{array}{l}\text { - Maturity of conventional film casting } \\
\text { - Advancements ongoing in film casting, sheet } \\
\text { extrusion processes } \\
\text { - Regulatory impedances and uncertainty on } \\
\text { application due to unavailability of marketed } \\
\text { products } \\
\text { - Advancement of other surrogate fields ongoing for } \\
\text { individualized drug delivery eg. microfluidics, } \\
\text { dripping }\end{array}$ \\
\hline
\end{tabular}




\section{Polymeric ODF manufacturing by drop printing of molten ink formulation}

An approach similar to solvent-based (inkjet) printing for molten ink formulations is being devolved by Reklaitis and colleagues (Reklaitis, n.d.; Hirshfield et al., 2014) . Briefly, dropon-demand (DoD) printing technology is used to deposit molten formulation drops onto edible films. As an example, a recent case study published by Içten and colleagues is discussed (Içten et al., 2015a). Hot melts of naproxen (NAP) and poly (ethylene glycol) (PEG) or Pluronic $\AA$ were deposited with high dosing reproducibility onto HPMC-PEG 400 films. The process was developed in a semi-continuous operation mode. The organic solvent, API and polymer mixture was heated above its melting temperature and pumped through a DoD positive displacement pump. Temperature regulation was extended to the whole system including reservoirs, tubing, and nozzles. The drops were formed by the application of a pressure pulse upstream of the nozzle. In order to monitor the process, a camera equipped with motor sensors was able to estimate accurately the drop volume and the formation of satellite drops. After ink deposition, the formulation was assessed for its drug dissolution profile, reproducibility of dosage amounts, and physical form of NAP after printing.

The drug remained stable as no degradation peaks were detected.. X-Ray diffraction studies of showed that the NAP completely crystallized into the starting polymorphic form after deposition irrespective of formulation. The differences in the rate and extent of dissolution were associated to the polymeric carrier in two formulations. Drug dosage was consistent with the number of drops deposited at a low standard deviation. The high drop placement precision and highly controllable drop volume makes this technology suitable for the manufacturing of solid dosage forms for high potency drugs. The same group has also recently published on the process control and automation of DoD and the impact of melt solidification temperature, drop size and cooling rates in tailoring the melt-crystallisation 
tendency and in vitro dissolution behaviour of the melt-printed formulations (H. Hsu et al., 2015; Içten et al., 2015b, 2014). Prasad and colleagues recently used an electrostatic API or formulation powder deposition approach on to the substrate film followed by subsequent coalesce of powder with substrate in infrared oven (Prasad et al., 2015).

\section{Conclusion and future direction}

There is an increasing need for patient-centric formulations tailored for patient populations. The drug product design should ensure safety and efficacy as well as patient acceptability and usability. The ODF platform is a promising technology broadening the options for developing and manufacturing drug products for distinct patient populations. The existing technical manufacturing challenges and the lack of consensus on the empirical definition of patient acceptability criteria limit the broader industrial investment on conventional and novel ODF technology. Collaborative approach of pharmaceutical industry, regulatory bodies and academia is required to pave the way towards optimising the quality attributes of ODFs both for industrial manufacturing and patient acceptability. In this perspective, future studies will be needed with the purpose of addressing the technical issues encountered by industrial partners and simultaneously match the geriatric-centric acceptability criteria suggested in this manuscript.

\section{References}

Abdelbary, A., Bendas, E.R., Ramadan, A.A., Mostafa, D.A., 2014. Pharmaceutical and pharmacokinetic evaluation of a novel fast dissolving film formulation of flupentixol dihydrochloride. AAPS PharmSciTech 15, 1603-10. doi:10.1208/s12249-014-0186-8

Alayoubi, A., Haynes, L., Patil, H., Daihom, B., Helms, R., Almoazen, H., 2016. Development of a fast dissolving film of epinephrine hydrochloride as a potential anaphylactic treatment for pediatrics. Pharm. Dev. Technol. 1-5. doi:10.3109/10837450.2015.1131715

Alomari, M., Mohamed, F.H., Basit, A.W., Gaisford, S., 2014. Personalised dosing: printing a dose of one's own medicine. Int. J. Pharm. 494, 568-77. 
doi:10.1016/j.ijpharm.2014.12.006

Ashraf, M., Sayeed, V.A., 2014. Considerations in Developing Sublingual Tablets - An Overview. Pharm. Technol. Eur. 26, 33.

Bala, R., Pawar, P., Khanna, S., Arora, S., 2013. Orally dissolving strips: A new approach to oral drug delivery system. Int. J. Pharm. Investig. 3, 67-76. doi:10.4103/2230973X.114897

Boehm, R.D., Daniels, J., Stafslien, S., Nasir, A., Lefebvre, J., Narayan, R.J., 2015. Polyglycolic acid microneedles modified with inkjet-deposited antifungal coatings. Biointerphases 10, 11004. doi:10.1116/1.4913378

Boehm, R.D., Miller, P.R., Daniels, J., Stafslien, S., Narayan, R.J., 2014. Inkjet printing for pharmaceutical applications. Mater. Today 17, 247-252. doi:10.1016/j.mattod.2014.04.027

Borges, A.F., Silva, C., Coelho, J.F.J., Simões, S., 2015a. Oral films: current status and future perspectives: I - Galenical development and quality attributes. J. Control. Release 206, 1-19. doi:10.1016/j.jconrel.2015.03.006

Borges, A.F., Silva, C., Coelho, J.F.J., Simões, S., 2015b. Oral films: current status and future perspectives II - Intellectual property, technologies and market needs. J. Control. Release 206, 108-21. doi:10.1016/j.jconrel.2015.03.012

Cheow, W.S., Kiew, T.Y., Hadinoto, K., 2015. Combining inkjet printing and amorphous nanonization to prepare personalized dosage forms of poorly-soluble drugs. Eur. J. Pharm. Biopharm. 96, 314-21. doi:10.1016/j.ejpb.2015.08.012

Choudhary, D.R., Patel, V.A., Chhalotiya, U.K., Patel, H. V, Kundawala, A.J., 2013. Comparative pharmacokinetic studies of fast dissolving film and oral solution of ondansetron in rats. Curr. Drug Deliv. 10, 696-700.

Chowdhury, A.U., Dettmar, C.M., Sullivan, S.Z., Zhang, S., Jacobs, K.T., Kissick, D.J., Maltais, T., Hedderich, H.G., Bishop, P.A., Simpson, G.J., 2014. Kinetic trapping of metastable amino acid polymorphs. J. Am. Chem. Soc. 136, 2404-12. doi: $10.1021 / \mathrm{ja} 410293 \mathrm{p}$

Clarke, A.J., Doughty, D.G., Fiesser, F.H., Wagner, D.S., 2012. Apparatus for producing a pharmaceutical product. US $8252234 \mathrm{~B} 2$.

Daly, R., Harrington, T.S., Martin, G.D., Hutchings, I.M., 2015. Inkjet printing for pharmaceutics - A review of research and manufacturing. Int. J. Pharm. 494, 554-67. doi:10.1016/j.ijpharm.2015.03.017

Deegan, R.D., Bakajin, O., Dupont, T.F., Huber, G., Nagel, S.R., Witten, T.A., 1997. Capillary flow as the cause of ring stains from dried liquid drops 389, 827-829. doi: $10.1038 / 39827$

Derby, B., 2010. Inkjet printing of functional and structural materials: fluid property requirements, feature stability, and resolution. Annu. Rev. Mater. Res. 40, 395-414. doi:10.1146/annurev-matsci-070909-104502

Di Risio, S., Yan, N., 2007. Piezoelectric ink-jet printing of horseradish peroxidase: effect of ink viscosity modifiers on activity. Macromol. Rapid Commun. 28, 1934-1940. doi:10.1002/marc.200700226 
Edinger, M., Bar-Shalom, D., Rantanen, J., Genina, N., 2017. Visualization and nondestructive quantification of inkjet-printed pharmaceuticals on different substrates using Raman spectroscopy and Raman chemical imaging. Pharm. Res. doi:10.1007/s11095017-2126-2

Garsuch, V., Breitkreutz, J., 2010. Comparative investigations on different polymers for the preparation of fast-dissolving oral films. J. Pharm. Pharmacol. 62, 539-45. doi:10.1211/jpp/62.04.0018

Genina, N., Fors, D., Palo, M., Peltonen, J., Sandler, N., 2013a. Behavior of printable formulations of loperamide and caffeine on different substrates--effect of print density in inkjet printing. Int. J. Pharm. 453, 488-97. doi:10.1016/j.ijpharm.2013.06.003

Genina, N., Fors, D., Vakili, H., Ihalainen, P., Pohjala, L., Ehlers, H., Kassamakov, I., Haeggström, E., Vuorela, P., Peltonen, J., Sandler, N., 2012. Tailoring controlled-release oral dosage forms by combining inkjet and flexographic printing techniques. Eur. J. Pharm. Sci. 47, 615-23. doi:10.1016/j.ejps.2012.07.020

Genina, N., Kolakovic, R., Palo, M., Fors, D., Juvonen, H., Lhalainen, P., Peltonen, J., Sandler, N., 2013b. Fabrication of printed drug-delivery systems, in: International Conference on Digital Printing Technologies. pp. 236-238.

Grimsrud, K.N., Sherwin, C.M.T., Constance, J.E., Tak, C., Zuppa, A.F., Spigarelli, M.G., Mihalopoulos, N.L., 2015. Special population considerations and regulatory affairs for clinical research. Clin. Res. Regul. Aff. 32, 47-56. doi:10.3109/10601333.2015.1001900

Gupta, A., Epstein, J.B., Sroussi, H., 2006. Hyposalivation in elderly patients. J Can Dent Assoc 72, 841-6.

Hilgert, E., De Beer, T., Sandler, N., Wickström, H., 2014. Development of pharmaceutical ink formulations with silica nanoparticles for inkjet printing. Ghent University.

Hirshfield, L., Giridhar, A., Taylor, L.S., Harris, M.T., Reklaitis, G. V, 2014. Dropwise additive manufacturing of pharmaceutical products for solvent-based dosage forms. J. Pharm. Sci. 103, 496-506. doi:10.1002/jps.23803

Hoath, S., Martin, G., Tuladhar, T., Mackley, M., Hutchings, I., 2008. Links between fluid rheology and drop-on-demand jetting and printability. NIP Digit. Fabr. Conf. 2008 Int. Conf. Digit. Print. Technol. 130-33.

Hoath, S.D., Jung, S., Hsiao, W.-K., Hutchings, I.M., 2012. How PEDOT:PSS solutions produce satellite-free inkjets. Org. Electron. 13, 3259-3262. doi:10.1016/j.orgel.2012.10.004

Hoffmann, E.M., Breitenbach, A., Breitkreutz, J., 2011b. Advances in orodispersible films for drug delivery. Expert Opin. Drug Deliv. 8, 299-316. doi: $10.1517 / 17425247.2011 .553217$

Hsu, H.-Y., Harris, M.T., Toth, S., Simpson, G.J., 2015. Drop printing of pharmaceuticals: Effect of molecular weight on PEG coated-naproxen/PEG 3350 solid dispersions. AIChE J. 61, 4502-4508. doi:10.1002/aic.14979

Hsu, H., Adigun, O.O., Taylor, L.S., Murad, S., Harris, M.T., 2015. Crystallization of acetaminophen on chitosan films blended with different acids. Chem. Eng. Sci. 126, 19. doi:10.1016/j.ces.2014.10.046 
Içten, E., Giridhar, A., Taylor, L.S., Nagy, Z.K., Reklaitis, G. V., 2015a. Dropwise additive manufacturing of pharmaceutical products for melt-based dosage forms. J. Pharm. Sci. 104, 1641-9. doi:10.1002/jps.24367

Içten, E., Nagy, Z.K., Reklaitis, G. V., 2015b. Modelling of crystallization of solid oral drug forms in a dropwise additive manufacturing system, 12th International Symposium on Process Systems Engineering and 25th European Symposium on Computer Aided Process Engineering - Computer Aided Chemical Engineering, Computer Aided Chemical Engineering. Elsevier. doi:10.1016/B978-0-444-63576-1.50060-1

Içten, E., Nagy, Z.K., Reklaitis, G. V., 2014. Supervisory control of a drop on demand minimanufacturing system for pharmaceuticals, 24th European Symposium on Computer Aided Process Engineering - Computer Aided Chemical Engineering, Computer Aided Chemical Engineering. Elsevier. doi:10.1016/B978-0-444-63456-6.50090-9

Illangakoon, U.E., Gill, H., Shearman, G.C., Parhizkar, M., Mahalingam, S., Chatterton, N.P., Williams, G.R., 2014. Fast dissolving paracetamol/caffeine nanofibers prepared by electrospinning. Int. J. Pharm. 477, 369-79. doi:10.1016/j.ijpharm.2014.10.036

Irfan, M., Rabel, S., Bukhtar, Q., Qadir, M.I., Jabeen, F., Khan, A., 2015. Orally disintegrating films: A modern expansion in drug delivery system. Saudi Pharm. J. In press. doi:10.1016/j.jsps.2015.02.024

Karki, S., Kim, H., Na, S.J., Shin, D., Jo, K., Lee, J., 2016. Thin films as an emerging platform for drug delivery. Asian J. Pharm. Sci. doi:10.1016/j.ajps.2016.05.004

Krampe, R., Visser, J.C., Frijlink, H.W., Breitkreutz, J., Woerdenbag, H.J., Preis, M., 2015. Oromucosal film preparations: points to consider for patient centricity and manufacturing processes. Expert Opin. Drug Deliv. 8, 1-14. doi:[Epub ahead of print]

Kuang, M., Wang, L., Song, Y., 2014. Controllable printing droplets for high-resolution patterns. Adv. Mater. 26, 6950-8. doi:10.1002/adma.201305416

Lind, J., Kälvemark Sporrong, S., Kaae, S., Rantanen, J., Genina, N., 2016. Social aspects in additive manufacturing of pharmaceutical products. Expert Opin. Drug Deliv. 1-10. doi:10.1080/17425247.2017.1266336

López-Verdín, S., Andrade-Villanueva, J., Zamora-Perez, A.L., Bologna-Molina, R., Cervantes-Cabrera, J.J., Molina-Frechero, N., 2013. Differences in salivary flow level, xerostomia, and flavor alteration in mexican HIV patients who did or did not receive antiretroviral therapy. AIDS Res. Treat. 2013, 613278. doi:10.1155/2013/613278

Moritz, C., 2006. Films that dissolve diagnostics manufacturers' needs. Med. Des. Technol. 10,11 .

Nallan, H.C., Sadie, J.A., Kitsomboonloha, R., Volkman, S.K., Subramanian, V., 2014. Systematic design of jettable nanoparticle-based inkjet inks: rheology, acoustics, and jettability. Langmuir 30, 13470-7. doi:10.1021/la502903y

Newman, J.A., Schmitt, P.D., Toth, S.J., Deng, F., Zhang, S., Simpson, G.J., 2015. Parts per million powder X-ray diffraction. Anal. Chem. 87, 10950-5. doi:10.1021/acs.analchem.5b02758

Palem, C.R., Dudhipala, N.R., Battu, S.K., Repka, M.A., Rao Yamsani, M., 2016. Development, optimization and in vivo characterization of domperidone-controlled release hot-melt-extruded films for buccal delivery. Drug Dev. Ind. Pharm. 42, 473-484. 
doi:10.3109/03639045.2015.1104346

Palem, C.R., Kumar Battu, S., Maddineni, S., Gannu, R., Repka, M.A., Yamsani, M.R., 2013. Oral transmucosal delivery of domperidone from immediate release films produced via hot-melt extrusion technology. Pharm. Dev. Technol. 18, 186-195. doi: $10.3109 / 10837450.2012 .693505$

Palo, M., Kogermann, K., Genina, N., Fors, D., Peltonen, J., Heinämäki, J., Sandler, N., 2016. Quantification of caffeine and loperamide in printed formulations by infrared spectroscopy. J. Drug Deliv. Sci. Technol. doi:10.1016/j.jddst.2016.02.007

Park, J.-B., Prodduturi, S., Morott, J., Kulkarni, V.I., Jacob, M.R., Khan, S.I., Stodghill, S.P., Repka, M.A., 2015. Development of an antifungal denture adhesive film for oral candidiasis utilizing hot melt extrusion technology. Expert Opin. Drug Deliv. 12, 1-13. doi: $10.1517 / 17425247.2014 .949235$

Peck, R.W., 2015. The right dose for every patient: a key step for precision medicine. Nat. Rev. Drug Discov. 15, 145-146. doi:10.1038/nrd.2015.22

Planchette, C., Pichler, H., Wimmer-Teubenbacher, M., Gruber, M., Gruber-Woelfler, H., Mohr, S., Tetyczka, C., Hsiao, W.-K., Paudel, A., Roblegg, E., Khinast, J., 2015. Printing medicines as orodispersible dosage forms: Effect of substrate on the printed micro-structure. Int. J. Pharm. [Epub ahea. doi:10.1016/j.ijpharm.2015.10.054

Prasad, L.K., Keen, J.M., LaFountaine, J.S., Maincent, J., Williams, R.O., McGinity, J.W., 2015. Electrostatic powder deposition to prepare films for drug delivery. J. Drug Deliv. Sci. Technol. 30, 501-510. doi:10.1016/j.jddst.2015.08.011

Preis, M., 2015. Orally disintegrating films and mini-tablets-innovative dosage forms of choice for pediatric use. AAPS PharmSciTech 16, 234-41. doi:10.1208/s12249-0150313-1

Preis, M., Breitkreutz, J., Sandler, N., 2015. Perspective: Concepts of printing technologies for oral film formulations. Int. J. Pharm. doi:10.1016/j.ijpharm.2015.02.032

Raijada, D., Genina, N., Fors, D., Wisaeus, E., Peltonen, J., Rantanen, J., Sandler, N., 2013. A step toward development of printable dosage forms for poorly soluble drugs. J. Pharm. Sci. 102, 3694-704. doi:10.1002/jps.23678

Rantanen, J., Khinast, J., 2015. The future of pharmaceutical manufacturing sciences. J. Pharm. Sci. 104, 3612-3638. doi:10.1002/jps.24594

Reis, N., Ainsley, C., Derby, B., 2005. Ink-jet delivery of particle suspensions by piezoelectric droplet ejectors. J. Appl. Phys. 97, 094903/1-094903/6. doi:10.1063/1.1888026

Reklaitis, R., n.d. Developments in drop on demand technology for dosage formation [WWW Document]. NSF Eng. Res. Cent. Struct. Org. Pariculate Syst. URL http://www.nipte.org/sites/default/files//documents/Developments in Drop on Demand Technology for Dosage Formation - Rex Reclaitis \%26 Michael Harris.pdf (accessed 3.17.16).

Repka, M.A., Prodduturi, S., Stodghill, S.P., 2003. Production and characterization of hotmelt extruded films containing clotrimazole. Drug Dev. Ind. Pharm. 29, 757-765. doi:10.1081/DDC-120021775

Sandler, N., Kassamakov, I., Ehlers, H., Genina, N., Ylitalo, T., Haeggstrom, E., 2014. Rapid 
interferometric imaging of printed drug laden multilayer structures. Sci. Rep. 4, 4020. doi:10.1038/srep04020

Sandler, N., Määttänen, A., Ihalainen, P., Kronberg, L., Meierjohann, A., Viitala, T., Peltonen, J., 2011. Inkjet printing of drug substances and use of porous substratestowards individualized dosing. J. Pharm. Sci. 100, 3386-95. doi:10.1002/jps.22526

Schmitt, P.D., Trasi, N.S., Taylor, L.S., Simpson, G.J., 2015. Finding the needle in the haystack: characterization of trace crystallinity in a commercial formulation of paclitaxel protein-bound particles by raman spectroscopy enabled by second harmonic generation microscopy. Mol. Pharm. 12, 2378-83. doi:10.1021/acs.molpharmaceut.5b00065

Siddiqui, M.D.N., Garg, G., Sharma, P.K., 2010. A short review on "A novel approach in oral fast dissolving drug delivery system and their patents." Adv. Biol. Res. (Rennes). 5, 291-303.

Singh, M., Haverinen, H.M., Dhagat, P., Jabbour, G.E., 2010. Inkjet printing-process and its applications. Adv. Mater. 22, 673-85. doi:10.1002/adma.200901141

Slavkova, M., Breitkreutz, J., 2015. Orodispersible drug formulations for children and elderly. Eur. J. Pharm. Sci. 75, 2-9. doi:10.1016/j.ejps.2015.02.015

Soutari, N., Buanz, A.B.M., Gul, M.O., Tuleu, C., Gaisford, S., 2012. Quantifying crystallisation rates of amorphous pharmaceuticals with dynamic mechanical analysis (DMA). Int. J. Pharm. 423, 335-40. doi:10.1016/j.ijpharm.2011.11.010

Sowade, E., Mitra, K.Y., Ramon, E., Martinez-Domingo, C., Villani, F., Loffredo, F., Gomes, H.L., Baumann, R.R., 2016. Up-scaling of the manufacturing of all-inkjetprinted organic thin-film transistors: Device performance and manufacturing yield of transistor arrays. Org. Electron. 30, 237-246. doi:10.1016/j.orgel.2015.12.018

Stegemann, S., Ecker, F., Maio, M., Kraahs, P., Wohlfart, R., Breitkreutz, J., Zimmer, A., Bar-Shalom, D., Hettrich, P., Broegmann, B., 2010. Geriatric drug therapy: neglecting the inevitable majority. Ageing Res. Rev. 9, 384-98. doi:10.1016/j.arr.2010.04.005

Stegemann, S., Ternik, R.L., Onder, G., Khan, M.A., Van Riet-Nales, D.A., 2016. Defining patient centric pharmaceutical drug product design. AAPS J. doi:10.1208/s12248-0169938-6

Stokes, J.R., Boehm, M.W., Baier, S.K., 2013. Oral processing, texture and mouthfeel: from rheology to tribology and beyond. Curr. Opin. Colloid Interface Sci. 18, 349-359. doi:10.1016/j.cocis.2013.04.010

Stringer, J., Derby, B., 2010. Formation and stability of lines produced by inkjet printing. Langmuir 26, 10365-72. doi:10.1021/la101296e

Tucker, G., DeSilva, B., Dressman, J., Ito, M., Kumamoto, T., Mager, D., Mahler, H.-C., Maitland-van der Zee, A.H., Pauletti, G.M., Sasaki, H., Shah, V., Tang, D., Ward, M., 2016. Current challenges and potential opportunities for the pharmaceutical sciences to make global Impact: an FIP perspective. J. Pharm. Sci. In press. doi:10.1016/j.xphs.2015.12.001

Vakili, H., Kolakovic, R., Genina, N., Marmion, M., Salo, H., Ihalainen, P., Peltonen, J., Sandler, N., 2015. Hyperspectral imaging in quality control of inkjet printed personalised dosage forms. Int. J. Pharm. 483, 244-9. 
doi:10.1016/j.ijpharm.2014.12.034

Ventola, C.L., 2014. Medical applications for 3D printing: current and projected uses. P T 39, 704-11.

Visser, J.C., Woerdenbag, H.J., Hanff, L.M., Frijlink, H.W., 2016. Personalized medicine in pediatrics: the clinical potential of orodispersible films. Aaps pharmscitech 104, 1292 1300. doi:10.1208/s12249-016-0515-1

Wickström, H., Palo, M., Rijckaert, K., Kolakovic, R., Nyman, J.O., Määttänen, A., Ihalainen, P., Peltonen, J., Genina, N., de Beer, T., Löbmann, K., Rades, T., Sandler, N., 2015. Improvement of dissolution rate of indomethacin by inkjet printing. Eur. J. Pharm. Sci. 75, 90-100. doi:10.1016/j.ejps.2015.03.009

Williams, H.D., Trevaskis, N.L., Charman, S.A., Shanker, R.M., Charman, W.N., Pouton, C.W., Porter, C.J.H., 2013. Strategies to address low drug solubility in discovery and development. Pharmacol. Rev. 65, 315-499. doi:10.1124/pr.112.005660

Zhang, J., Zhao, S., Zhu, Y., Huang, Y., Zhu, M., Tao, C., Zhang, C., 2014. Threedimensional printing of strontium-containing mesoporous bioactive glass scaffolds for bone regeneration. Acta Biomater. 10, 2269-81. doi:10.1016/j.actbio.2014.01.001 